\title{
Bronchopulmonary infection with Pseudomonas aeruginosa in patients infected with human immunodeficiency virus
}

\author{
Nabeel J Ali, David Kessel, Robert F Miller
}

\begin{abstract}
Background-Pseudomonas aeruginosa infection is uncommon in HIV infected patients and is usually nosocomially acquired and associated with risk factors such as neutropenia or central lines. We have recently noted an increase in the number of respiratory isolates of $P s$ aeruginosa in hospitalised HIV positive patients and sought to describe the clinical correlates of this observation.
\end{abstract}

Methods-A retrospective case notes review of HIV positive patients admitted to a specialist unit for respiratory investigations from January 1989 to December 1993 was undertaken in order to identify those with Ps aeruginosa respiratory infection and to describe associated risk factors, patterns of presentation and radiographic abnormalities.

Results-Of 617 patients admitted 38 (6\%) had Ps aeruginosa respiratory infection (notes were incomplete in 1 patient). All patients had advanced HIV disease; median CD4 $=0.02 \times 10^{9} / 1$. Two distinct presentations were seen; 9 patients had a fulminant course as part of a sepsis syndrome, 28 patients had an indolent presentation (18 had a single episode and 10 relapsed on one or more occasions, despite successful treatment of the initial episode). Infection was community acquired in 24 patients. Many patients had risk factors traditionally associated with $P$ s aeruginosa including neutropenia or indwelling central venous catheters, but 13 had no obvious risk factor. Most patients were receiving systemic pneumocystis prophylaxis and/or broad spectrum antibiotics; 20 had coexistent symptomatic sinus disease. A wide variety of chest radiographic abnormalities were seen including interstitial shadowing, mimicking pneumocystis pneumonia in 12 patients, lobar pneumonia in 2 and bronchial wall thickening in 13 patients.

Conclusions-Ps aeruginosa respiratory infection occurs with increased frequency in patients with advanced HIV disease; in a significant proportion infection is community acquired. Although recognised risk factors were present in two thirds of patients it appears that advanced HIV immunosuppression, use of systemic pneumocystis prophylaxis and/or broad spectrum antibiotics and sinus disease are important risk factors. The diagnosis should be considered in patients with advanced HIV disease who present with new respiratory symptoms.

(Genitourin Med 1995;71:73-77)

Keywords: Pseudomonas aeruginosa; HIV; Bronchopneumonia

\section{Introduction}

Bacterial infections including pneumonia are seen with increasing frequency in patients infected with the human immunodeficiency virus (HIV). ${ }^{1}$ Community-acquired bacterial pneumonias are most often caused by encapsulated organisms such as Streptococcus pneumoniae and Haemophilus influenzae, ${ }^{2}$ whereas nosocomial pneumonia is frequently due to Staphylococcus aureus ${ }^{3}$ or Gram negative organisms and frequently occurs in patients with advanced HIV disease, many of whom are neutropenic ${ }^{4}$ or have indwelling central venous catheters. ${ }^{5}$ Pneumonia in this setting is often associated with septicaemia and has high mortality. ${ }^{4} 6$

Recently twelve patients with advanced HIV disease have been described with an indolent community-acquired Pseudomonas aeruginosa bronchopulmonary infection without associated risk factors such as neutropenia or central venous line, a low mortality rate and high rate of relapse following treatment, a clinical picture very similar to that seen in patients with cystic fibrosis. ${ }^{7}$ We undertook this study to determine the incidence of Ps aeruginosa bronchopulmonary infection and its clinical features in HIV seropositive patients admitted to a specialist unit for investigations of respiratory symptoms.

\section{Methods}

Consecutive HIV antibody positive patients admitted to a specialist inpatient unit for respiratory investigations from January 1989 until December 1993 were studied. Potential cases of bronchopulmonary infection with $P S$ aeruginos $a$ were identified in two ways. Firstly by searching the computerised records of the Department of Medical Microbiology, University College London Hospitals, for all isolates of $P s$ aeruginosa in sputum, induced sputum, bronchoalveolar lavage fluid, pleural fluid and blood, and secondly by searching the computerised records of inpatient discharge summaries for the word pseudomonas.

From the records of each patient with a 
positive respiratory isolate of Ps aeruginosa we obtained the following data: (1) Age, risk factors for HIV infection, previous manifestations of HIV disease, smoking history and presence of underlying medical conditions (including respiratory disease), (2) Type and use of pneumocystis prophylaxis, other antibiotics, zidovudine, ganciclovir, glucocorticoids and chemotherapy, (3) Presence of absence of an indwelling central venous line, (4) Presence of symptomatic sinus disease, (5) Symptoms and signs, (6) Peripheral blood white blood cell count and neutrophil count (neutropenia was defined as $<1.5 \times 10^{9} / 1$ ), CD4 lymphocyte count (CD4 lymphocyte counting became available in mid 1991 and was available in 33 patients), (7) The site(s) of isolation of Ps aeruginosa, (8) Whether infection was nosocomial or community-acquired. Nosocomial infection was defined as that developing in a patient who had been treated in hospital or who had lived in a hospice within the previous two months, (9) Clinical outcome including complications, death and recurrence of infection.

Chest radiographs were reviewed (by DK who was blind to the clinical diagnosis). The pattern (alveolar or interstitial) of parenchymal abnormality, the presence of pleural effusion, cavitation and bronchial wall thickening was noted. Relapse was defined as onset of new respiratory symptoms, with or without new radiographic abnormalities, together with isolation of $P s$ aeruginosa in a respiratory isolate.

\section{Results}

During the period of the study 617 patients were admitted for investigation of respiratory symptoms. Thirty eight of them (6\%) had Ps aeruginosa respiratory infection; patient records were incomplete in one patient, and therefore we report the results in thirty seven patients. Thirty five patients were men, 34 were Caucasian homosexuals and one was an Indian recipient of a contaminated blood transfusion; the two women were heterosexual Africans. None used intravenous drugs and 15 were cigarette smokers, three were exsmokers. Their mean age was 35 years (range 25-56), none had diabetes mellitus nor heart

Table 1 Clinical and laboratory features of patients with Pseudomonas aeruginosa bronchopulmonary infection

\begin{tabular}{|c|c|c|c|}
\hline \multirow[b]{2}{*}{$\begin{array}{l}\text { Clinical laboratory } \\
\text { features }\end{array}$} & \multirow[b]{2}{*}{$\begin{array}{l}\text { Fulminant } \\
(n=9)\end{array}$} & \multicolumn{2}{|l|}{ Indolent } \\
\hline & & $\begin{array}{l}\text { Single episode } \\
(n=18)\end{array}$ & $\begin{array}{l}\text { Relapsing } \\
(n=10)\end{array}$ \\
\hline Community acquired & 5 & 10 & 9 \\
\hline PCP prophylaxis & 8 & 10 & 9 \\
\hline Other antibiotics & 3 & 7 & 5 \\
\hline Zidovudine & 3 & 5 & 5 \\
\hline Ganciclovir & 3 & 0 & 1 \\
\hline Glucocorticoids & 1 & 2 & 1 \\
\hline Central venous catheter & 4 & 0 & 1 \\
\hline $\begin{array}{l}\text { WBC } \\
\text { range (mean) } \times 10^{9} 1\end{array}$ & $10-20 \cdot 0(4 \cdot 9)$ & $1 \cdot 1-8 \cdot 0(4 \cdot 2)$ & $1.4-7.0(2 \cdot 8)$ \\
\hline $\begin{array}{l}\text { Neutrophils } \\
\text { range }(\text { mean }) \times 10^{9} 1\end{array}$ & $0.7-16.0(3.7)$ & $0.5-6.3(2.8)$ & $0.9-4.8(1.8)$ \\
\hline Neutropenia & 4 & 4 & 6 \\
\hline Sinus disease & 3 & 10 & 7 \\
\hline Survived episode & 1 & 4 & 10 \\
\hline
\end{tabular}

$\mathrm{PCP}=$ Pneumocystis carinii pneumonia disease; four patients had bronchiectasis (one since childhood, one following tuberculosis and two following previous episodes of Pneumocystis carinii pneumonia).

All but two patients had prior AIDS diagnoses including $P$ carinii pneumonia in 16 , of these two also had Kaposi's sarcoma, two also had Kaposi's sarcoma and cytomegalovirus (CMV) retinitis, and two disseminated Mycobacterium avium-intracellulare infection, (one of these also had CMV retinitis). Seven others had Kaposi's sarcoma (three with $M$ avium-intracellulare), two had CMV retinitis and one had $M$ avium-intracellulare. All were profoundly immunosuppressed: median CD4 count was 0.02 (range $=0-0.16) \times 10^{9 / 1}$ (normal range $=0.35-2.2 \times 10^{9} 1 \mathrm{l}$. In four patients co-pathogens were identified; $M$ aviumintracellulare in three (two community acquired infection) and Streptococcus pneumoniae in one (nosocomial infection).

Twenty-seven patients were taking antipneumocystis prophylaxis; co-trimoxazole in 18 (eight of whom were also taking zidovudine), nebulised pentamidine in five (three were also taking zidovudine), dapsone in three and IV pentamidine in one. Two other patients were taking zidovudine but no prophylaxis. One patient was receiving chemotherapy for Kaposi's sarcoma (table 1).

All patients had symptoms of cough, dyspnoea and fever, 27 had sputum expectoration and 2 had haemoptysis. Two distinct clinical presentations were seen; firstly, nine patients had a fulminant course presenting with a sepsis syndrome. In five of these patients infection was community acquired. Ps aeruginosa was also isolated from blood in two patients, blood and pleural fluid in one patient and pleural fluid in another patient. Despite treatment with antipseudomonal antibiotics, eight patients died. Three of the four patients with nosocomially acquired infection were receiving broad spectrum antibiotics at the time of diagnosis (table 1).

Twenty eight patients had an indolent presentation with an onset of symptoms occurring over several days or weeks. Eighteen patients had a single episode of infection, but in 10 patients relapse occurred despite apparently successful treatment of the initial episode. Of the 18 patients with the single indolent episode infection was community acquired in 10.

Of eight with nosocomial infection four were receiving broad spectrum antibiotics for respiratory symptoms, one was receiving clindamycin for cerebral toxoplasmosis and one was receiving rifampicin, ciprofloxacin and clarithromycin for disseminated $M$ aviumintracellulare infection (table 1). Ps aeruginosa was also isolated from blood in one patient. Two patients were taking long term glucocorticoids, one as treatment for asthma the other to reduce oedema associated with disseminated Kaposi's sarcoma.

No antibiotic treatment for Ps aeruginosa was given to seven patients; all had advanced HIV disease with profound wasting and were terminally ill. Of 11 who received treatment 
Table 2 Chest radiographic appearances of patients with Pseudomonas aeruginosa bronchopulmonary infection

\begin{tabular}{|c|c|c|c|c|c|}
\hline \multirow[b]{2}{*}{ Patient group } & \multirow[b]{2}{*}{ Normal } & \multicolumn{4}{|l|}{ Abnormal } \\
\hline & & $\begin{array}{l}\text { Alveolar } \\
\text { consolidation }\end{array}$ & $\begin{array}{l}\text { Interstitial } \\
\text { infiltrates }\end{array}$ & $\begin{array}{l}\text { Bronchial } \\
\text { wall thickening }\end{array}$ & $\begin{array}{l}\text { Pleural } \\
\text { effusion }\end{array}$ \\
\hline $\begin{array}{c}\text { Fulminant } \\
(\mathrm{n}=9) \\
\text { Indolent }\end{array}$ & 1 & $5^{\star}$ & $3 \ddagger$ & 2 & 3 \\
\hline $\begin{array}{l}\text { Single episode } \\
\quad(\mathrm{n}=18)\end{array}$ & 6 & 4 & 9 & 10 & 2 \\
\hline $\begin{array}{l}\text { Relapsing } \\
\quad(n=10)\end{array}$ & 2 & $2^{\star}+$ & 5 & 1 & 0 \\
\hline
\end{tabular}

$\star_{\text {in }} 1$ patient in each group the alveolar consolidation was classically lobar.

tboth patients had cavitation.

$\neq 1$ patient had cavitation.

Figure 1a Chest radiograph. Diffuse interstitial shadowing mimicking Pneumocystis carinii pneumonia (fulminant presentation)

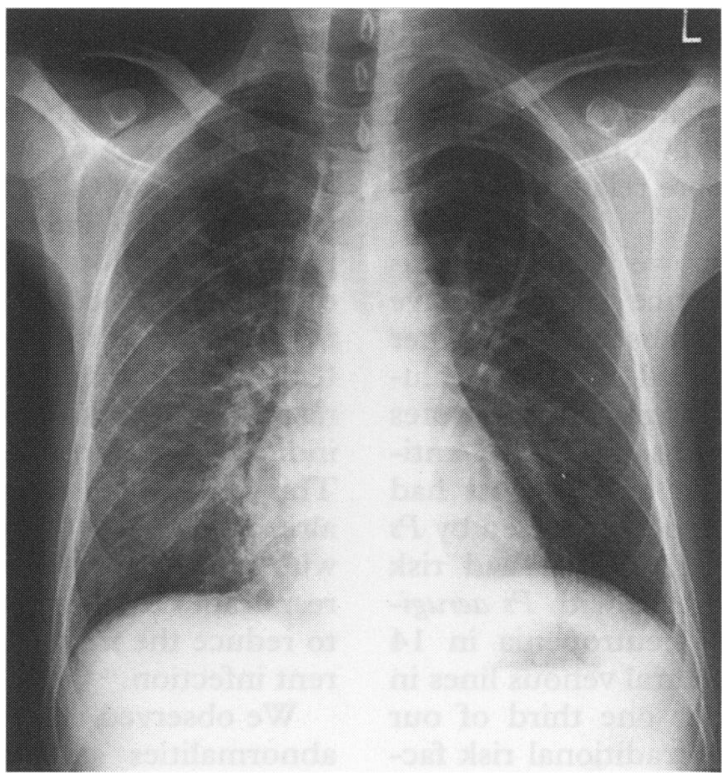

Figure $1 b$ Chest radiograph. Diffuse interstitial shadowing and a thick-walled cavitary lesion (arrows) (indolent presentation).

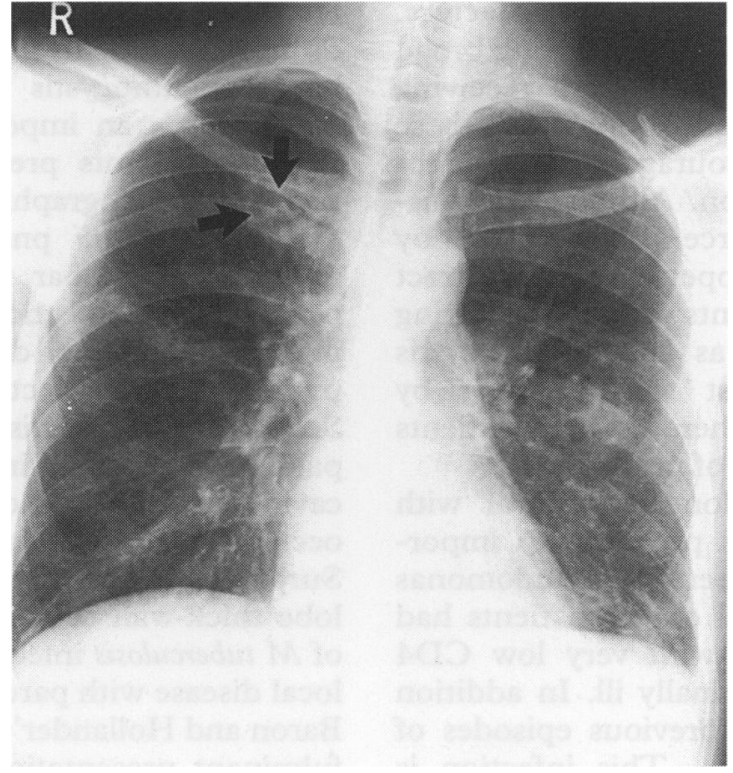

four survived for between two and 16 (months $($ mean $=7)$ ).

Ten patients had one or more relapses, with the first occurring between 1 and 6 months (median $=2$ ) after initial presentation. In nine of these the initial presentation was community acquired. Ps aeruginosa was also identified in blood in two patients and in pleural fluid in one other patient. Three patients were receiving rifampicin, ciprofloxacin and ethambutol as treatment for $M$ avium-intracellulare infection and two others were receiving broad spectrum antibiotics as outpatients as treatment for presumed "bronchitis". Survival in this group was 1-18 (median $=8$ ) months.

A wide range of radiographic abnormalities was seen in patients with both indolent and fulminant presentations (table 2, fig 1). Of note, of 11 patients with alveolar consolidation appearances were classically lobar in two. Twelve of 15 patients with interstitial infiltrates had changes typical of $P$ carinii pneumonia (and this diagnosis was suggested by DK). Thick walled cavitary lesions reminiscent of $M$ tuberculosis occurred in three patients. Bronchial wall thickening with or without dilatation, when present, was exclusively bilateral and lower lobe in site.

\section{Discussion}

In this study $P$ s aeruginosa infection accounted for $6 \%$ of respiratory admissions. Previous studies, from early in the AIDS epidemic, commented on the infrequency of $P s$ aeruginosa as a pathogen in HIV infected individuals and also on the fact that when it occurred it was almost always associated with traditional risk factors such as steroid and cytotoxic drug use and neutropenia ${ }^{4}$ and was usually a nosocomially acquired, rather than community acquired infection. Of 133 episodes of bacteraemia in patients with AIDS seven were due to Ps aeruginosa, all were nosocomial, and the majority of the patients were neutropenic. ${ }^{4}$ In another study $P$ s aeruginosa accounted for three of 44 episodes of community acquired bacteraemia, the lung was the source of bacteraemia in only one case. ${ }^{8}$ More recent studies report a higher incidence of Ps aeruginosa infection, again with traditionally associated risk factors. In one study eight of 79 episodes of septicaemia in patients with AIDS or ARC were caused by Ps aeruginosa; all infections were nosocomial, many patients had recently been in the Intensive Care Unit and had received broad spectrum antibiotics. ${ }^{9}$ Keilhofner et al reported seven cases of $P_{s}$ aeruginosa pneumonia in HIV infected patients, three had complicated pneumonia, one had empyema and two were bacteraemic; in two patients infection was community acquired. The one patient who survived lived a further 5 months and died without relapse. ${ }^{6}$ In a study from Milan 20 episodes of Ps aeruginosa pneumonia occurred in 428 patients with ARC or AIDS, none of whom were neutropenic and $70 \%$ of whom had CD4 counts $<0.04 \times 10^{9} / 1$. Infection was communityacquired in eight (three of whom had bacterial co-pathogens) none died but four relapsed, and was nosocomial in twelve (eleven had co-pathogens including $M$ avium-intracellulare in four, $M$ tuberculosis in three and cytomegalovirus in four (three also had pulmonary Kaposi's sarcoma)), one patient died and one patient relapsed. ${ }^{10}$ In another study, from the United States, 16 patients had Ps aeruginosa pneumonia, infection was commu- 
nity-acquired in 15 , four were neutropenic and mean CD4 count was $0.027 \times 10^{9} / 1$. Three patients died and four had relapses. ${ }^{11}$ In contrast to these studies Baron and Hollander $^{7}$ found that 16 of $1852 \mathrm{HIV}$ positive patients $(0.8 \%)$ attending an outpatient facility had Ps aeruginosa respiratory infection; all these patients had advanced HIV disease with a mean CD4 count of $0.025 \times 10^{9} / 1$. As in our study they described two distinct syndromes: four of their patients had a fulminant sepsis syndrome; in two infection was community acquired and two were neutropenic. One patient died and all three who survived relapsed one or more times with further episodes of Ps aeruginosa infection. Twelve patients had an indolent presentation, in all infection was community acquired and none were neutropenic. One patient died rapidly with Kaposi's sarcoma and 9 of the remaining 11 patients had one or more relapses of infection.

In our study, although we did not document serological evidence of invasive pulmonary disease, the absence of other pathogens in respiratory fluid and the resolution of symptoms and radiographic infiltrates in those treated with antipseudomonal antibiotics argue strongly that our patients had infection and were not simply colonised by $P_{s}$ aeruginosa. Several of our patients had risk factors traditionally associated with Ps aeruginosa infection, including neutropenia in 14 patients and indwelling central venous lines in five, but 13 patients, over one third of our study population, had no traditional risk factors. We looked for other associations, in the absence of traditionally associated risk factors. Fourteen of our patients were receiving broad spectrum antibiotics and 21 were receiving systemic anti-pneumocystis prophylaxis; these antibiotics may have encouraged development of pseudomonas infection. Although nebulisers are a recognised source of colonisation by pseudomonas of the upper respiratory tract the majority of our patients were not receiving nebulised pentamidine as anti-pneumocystis prophylaxis, in contrast to the study by Schuster and Norris, where $25 \%$ of patients were receiving this form of prophylaxis. ${ }^{11}$

In our patients prolonged survival with immunosuppression was probably an important factor for development of pseudomonas respiratory infection. All of our patients had late stage HIV disease with very low CD4 counts; some were terminally ill. In addition 16 of our patients had previous episodes of pneumocystis pneumonia. This infection is known to leave residual parenchymal lung damage and bronchiectasis following recovery, which might predispose to acquisition of pseudomonas. Four of our patients had bronchiectasis, two following $P$ carinii pneumonia. We did not perform CT of the chest in our patients but the finding of bronchial wall thickening on plain chest radiography in 13 patients suggests that we may have underestimated the frequency of bronchiectasis. In contrast to Baron and Hollander's study, ${ }^{7}$ where only one patient had sinus disease, $60 \%$ of our patients had symptomatic sinus disease. Sinus disease occurs with increased frequency in HIV infected individuals. ${ }^{12}$ In patients with advanced immunosuppression, and CD4 counts below $0.02 \times 10^{9} / 1$ the prevalence ranges from $1 \cdot 2 \%-6 \cdot 3 \%,{ }^{13}{ }^{14}$ and $P S$ aeruginosa is a common pathogen. ${ }^{1314}$ The prevalence of paranasal sinus abnormalities on cranial MRI is even higher; $15 \cdot 1 \%$ of patients with AIDS scanned for suspected intracranial pathology had evidence of moderate or severe sinus disease $(>75 \%$ obliteration of $\geqslant 1$ sinus). In addition there was a correlation between the degree of immunosuppression and the mean maximum mucosal thickening (indicative of chronic sinusitis) being worse in patients with AIDS than in HIV positive patients without AIDS. ${ }^{15}$

It is not clear whether the sinus is acting as a nidus for infection or whether there is diffuse abnormality of the respiratory tract mucosa in advanced HIV infection, which in some way permits infection with $P$ s aeruginosa. In view of the high rate of relapse in our patients and in those reported by Baron and Hollander it is tempting to suggest that long term maintenance anti-pseudomonal therapy might be indicated in those with relapsing infection. This form of therapeutic intervention is already used in non-HIV infected patients with cystic fibrosis who have pseudomonas respiratory tract infection and has been shown to reduce the frequency and severity of recurrent infection. ${ }^{16}$

We observed a wide variety of radiographic abnormalities associated with Ps aeruginosa infection in our patients, but no specific pattern was identified. Indeed many of those with diffuse interstitial changes were thought to have pneumocystis pneumonia. Thus Ps aeruginosa is an important diagnosis to consider in patients presenting with symptoms and chest radiographic abnormalities suggestive of $P$ carinii pneumonia. Similarly the finding of lobar consolidation means pseudomonas infection should be considered in the infectious differential diagnosis of pneumococcal infection. ${ }^{2}$ In the study by Schuster and Norris, ${ }^{11}$ six of their sixteen patients had lobar infiltrates and eight had cavitatory infiltrates on admission; cavitation occurred in a further three patients. Surprisingly three of our patients had upper lobe thick-wall cavitatory lesions reminiscent of $M$ tuberculosis infection, suggesting invasive local disease with parenchymal destruction. In Baron and Hollander's study all patients with a fulminant presentation had alveolar consolidation as did eight with an indolent course; only two patients had interstitial infiltrates and two had normal radiographs; none of the patients had cavitation. ${ }^{7}$ In the study from Milan $^{10}$ all 20 patients had interstitial infiltrates and eight also had cavitation and four had effusions. Interpretation of these findings is difficult because 14 patients had copathogen infections which may have contributed to the radiographic abnormalities. We found a high rate of complications in our patients, in contrast to previous reports which 
suggest empyema is uncommon in HIV infected patients with pyogenic bacterial pneumonia, ${ }^{17}$ empyema occurred in three of our patients.

In conclusion $P$ s aeruginosa respiratory infection is seen with increased frequency in patients with advanced HIV infection and AIDS. Two distinct patterns of presentation are seen, a fulminant sepsis syndrome and an indolent course. The radiographic abnormalities are non specific and may mimic pneumocystis pneumonia. In a significant proportion of patients infection is community acquired and in those with an indolent course there is a high rate of relapse. Although two thirds of patients have traditionally associated risk factors, in the remainder advanced HIV induced immunosuppression, use of systemic antipneumocystis prophylaxis or broad spectrum antibiotics, and symptomatic sinus disease appear to be important risk factors. Other factors that may be important, and require further study, include Ps aeruginosa-produced elastase, slime layer, exotoxin $\mathrm{A}$ and mucoid exopolysaccharide (MEP), and HIV-induced defects of intrapulmonary anti-elastase activity and failure of production of opsonic antibodies directed to $P s$ aeruginosa MEP. Ps aeruginosa should be considered in the differential diagnosis of new respiratory symptoms in patients with advanced HIV disease.

1 Pitkin AD, Grant AD, Foley NM, Miller RF. Changing patterns of respiratory disease in HIV positive patients in a referral centre in the United Kingdom between 1986-7 and 1990-1. Thorax 1993;48:204-7.
2 Miller RF, Foley NM, Kessel D, Jeffrey AA. Communityacquired lobar pneumonia in patients with HIV infection and AIDS. Thorax 1994;49:367-8.

3 Levine SJ, White DA, Fels AOS. The incidence and significance of Staphylococcus aureus in respiratory cultures from patients infected with the human immunodeficiency virus. Ann Rev Respir Dis 1990;141:89-93.

4 Witt DJ, Craven DE, McCabe WR. Bacterial infections in adult patients with the acquired immune deficiency syndrome (AIDS) and AIDS-related complex. $A m \mathcal{F} \mathrm{Med}$ 1987;82:900-6.

5 Nelson MR, Shanson DC, Barter GJ, Hawkins DA Gazzard BG. Pseudomonas septicaemia associated with HIV. AIDS 1991;5:761-3.

6 Kielhofner M, Atman RL, Hamill RH, Musher DM. Life threatening Pseudomonas aeruginosa infections in patients with human immunodeficiency virus disease. Clin Infect Dis 1992;14:403-11.

7 Baron AD, Hollander H. Pseudomonas aeruginosa bronchopulmonary infection in late human immunodefichopulmonary infection in late human immunodefi-

8 Krumholz HM, Sande MA, Lo B. Community acquired bacteraemia in patients with the acquired immunodeficiency syndrome (AIDS) and AIDS related complex. Am $\mathcal{F}$ Med 1987;82:900-6.

9 Shanson CD. Septicaemia in patients with AIDS. Tran Royal Soc Trop Med Hyg 1990;84 (suppl): 14-6.

10 Franzetti F, Cernuschi $M$, Esposito $R$, Moroni $M$ Pseudomonas infections in patients with AIDS and AIDS-related complex. F Intern Med 1992;231:437-43.

11 Schuster MG, Norris AH. Community-acquired Pseudomonas aeruginosa pneumonia in patients with HIV Pseudomonas aeruginosa pneumon
infection. AIDS 1994;8:1437-41.

12 Wallace JM, Rao AV, Glassroth J, et al. Respiratory illness in persons with human immunodeficiency virus infection. Am Rev Respir Dis 1993;148:1523-9.

13 Godofsky EW, Zinreich J, Armstrong $M$, Leslie JM, Weikel CS. Sinusitis in HIV-infected patients: a clinica and radiographic review. Am $\mathcal{F} M e d$ 1992;93:163-70.

14 Grant AD, VonSchoenberg MA, Grant HR, Miller RF Paranasal sinus disease in HIV antibody positive patients. Genitourin Med 1993;69:208-12.

15 Chong WK, Hall-Craggs MA, Wilkinson ID, et al. The prevalence of paranasal sinus disease in HIV infection and AIDS on cranial MR imaging. Clin Radiol 1993;47:166-9.

16 Hodson ME, Penketh RA, Batten JC. Aerosol Carbenicillin and gentamicin treatment of Pseudomonas aeruginosa infection in patients with cystic fibrosis. Lancet 1981;ii:1137-9.

17 Coker RJ. Empyema thoracis in AIDS. $f R$ Soc $M e d$ 1994;87:65-7. 